\title{
Indicador bibliométrico basado en el índice $\mathbf{h}$
}

\author{
Pablo Dorta-González*, María Isabel Dorta-González ${ }^{\star \star}$
}

Resumen: El índice $b$ se ha convertido en uno de los indicadores bibliométricos más empleados para estimar el éxito del trabajo realizado por un investigador y predecir el impacto de su producción en el futuro. Esto se debe principalmente a dos razones. En primer lugar, a su simplicidad, dado que se trata de un único indicador que combina producción e impacto, y puede ser determinado fácilmente por cualquier investigador. En segundo lugar, a que elimina los sesgos provocados por las colas de la distribución de citas. Sin embargo, este indicador presenta limitaciones al discriminar entre investigadores con diferentes hábitos de publicación, penalizando a aquellos más selectivos, que no destacan por el número de publicaciones pero sí por el alto número de citas recibidas, frente a los grandes productores. En este trabajo se proponen soluciones que consideran las citas de aquellos artículos que pueden contribuir, con una alta probabilidad, a incrementar el valor del índice $b$ en el futuro.

Palabras clave: Índice $b$, análisis de citas, indicadores bibliométricos, evaluación científica.

\section{Bibliometric indicator based on the $h$-index}

Abstract: The h index has become one of the most widely used bibliometric indicators for estimating the success of researchers and predicting the impact of their work in the future. This is mainly due to its simplicity, since it is a single indicator that combines production and impact, and can easily be determined by any researcher. It also eliminates the bias caused by the long tail of citation distribution. However, this indicator has limitations, in that it fails to discriminate between researchers with different publishing habits and, as a result, it penalizes those with a more selective output characterized by a relatively low number of frequently cited documents, as opposed to authors with a high number of publications. This paper proposes a solution that would take into consideration the citations of those publications with a bigh probability of increasing the future $\mathrm{h}$ index values.

Keywords: h-index, citation analysis, bibliometric indicators, scholarly evaluation.

* Departamento de Métodos Cuantitativos en Economía y Gestión. Universidad de Las Palmas de Gran Canaria, Gran Canaria, España. Correo-e: pdorta@dmc.ulpgc.es.

** Departamento de Estadística, Investigación Operativa y Computación. Universidad de La Laguna. Tenerife, España. Correo-e: isadorta@ull.es.

Recibido. 22-6-09; 2. ${ }^{a}$ versión: 29-9-09; aceptado: 21-10-09. 


\section{Introducción}

La investigación científica tiene gran influencia en el desarrollo económico y social de los países. Sin embargo, los recursos que pueden destinarse a esta actividad son limitados. La demanda de incremento en su financiación ha originado la necesidad de evaluar la actividad científica y sus resultados, siendo uno de los más directos las publicaciones científicas. El objetivo de los indicadores bibliométricos es el análisis cuantitativo y cualitativo de dichas publicaciones, a partir del número de artículos y el número de citas que estos reciben (impacto), y entre sus principales aplicaciones se encuentra el área de Política Científica.

Los indicadores clásicos, como el número de artículos, el número total de citas y el promedio de citas por artículo, de forma individual, no reflejan satisfactoriamente el éxito de la carrera profesional de un investigador. Además, no son robustos, en el sentido que se ven muy afectados tanto por los artículos poco citados como por aquellos anormalmente muy citados, no representativos de la actividad normal del investigador ya que podrían deberse a éxitos puntuales o grandes colaboraciones.

El índice $b$ (Hirsch, 2005) es un interesante indicador que estima el número de trabajos importantes publicados por un investigador, incrementando la exigencia a la vez que aumenta su valor. Se trata de un indicador robusto que considera al mismo tiempo aspectos cuantitativos y cualitativos o de visibilidad. Existe correlación entre el índice y el éxito de un investigador apreciado por sus pares (Hirsch, 2005) y tiene, además, valor predictivo del éxito futuro (Hirsch, 2007).

No obstante, también presenta algunas limitaciones. Aunque este indicador ha demostrado su utilidad para detectar a los investigadores más destacados en un área, existen evidencias empíricas de que no discrimina entre los situados en niveles intermedios, y penaliza a los selectivos frente a los grandes productores (Costas y Bordons, 2007a, b). Al comparar las carreras profesionales de dos científicos de un mismo campo, surgen dudas entre aquellos casos con valores similares de $b$ en los que las curvas de citas se cortan. Esto es debido a que algunos investigadores presentan más altos niveles de citas al comienzo de la curva y más bajos al final. En estos casos, es preciso establecer criterios que permitan discriminar que distribuciones de citas corresponden a carreras más consolidadas y constantes.

Por tanto, el problema que tratamos de resolver es el siguiente: ¿Cómo discriminar entre dos investigadores con valores similares de $b$ y curvas de citas que se cortan? En este trabajo se propone un indicador de entorno, basado en el índice $b$, que considera la zona intermedia de la distribución de citas.

El apartado 2 está dedicado a describir los principales indicadores bibliométricos empleados en la evaluación de la carrera profesional de un investigador, prestando especial atención a aquellos índices tipo $h$. En el apartado 3 se describen los indicadores de entorno y en el 4 se muestra un ejemplo de su aplicación práctica. Finalmente, el apartado 5 se dedica a conclusiones y consideraciones finales. 


\section{Indicadores bibliométricos para la evaluación de investigadores}

\subsection{Indicadores de producción e impacto}

Existe consenso en admitir que los artículos son el resultado más directo de la investigación, especialmente cuando se consideran aquellas revistas en las que existe un sistema de selección que garantiza la calidad y originalidad de los trabajos. Aunque el juicio de expertos ha demostrado ser el método más apropiado para valorar la calidad de las aportaciones al área de los artículos, el sistema presenta algunas limitaciones, como el carácter subjetivo y su elevado coste. En este contexto, los indicadores bibliométricos representan indicios objetivos de calidad, que pueden complementar al juicio de expertos.

Los indicadores bibliométricos más empleados se basan en el recuento de las publicaciones y las citas recibidas por los trabajos publicados, así como el impacto de las revistas de publicación. El número de publicaciones $\left(N_{p}\right)$, es un indicador cuantitativo que no valora la calidad del contenido. Es evidente que no todas las publicaciones tienen la misma importancia, ni suponen la misma contribución al avance científico. En la búsqueda de indicadores cualitativos, capaces de valorar estos aspectos, se han propuesto el número total de citas $\left(N_{c}\right)$ que reciben los trabajos, el promedio de citas por artículo $\left(n_{c}=N_{c} / N_{p}\right)$, el factor de impacto de la revista de publicación y la posición relativa de la revista en los listados temáticos, ordenados por factores de impacto, del Journal Citation Reports (JCR), atendiendo a la base de datos ISI-Web of Science de Thomson Reuters. Sin embargo, se ha observado que algunas publicaciones que reúnen criterios de calidad atendiendo a la opinión de expertos, apenas reciben citas. La razón es que el número de citas depende de su calidad, pero también de otras variables entre las que figura el prestigio del autor y/o la institución, la actualidad del tema, la lengua empleada y la revista de publicación, que condicionan la mayor o menor difusión del trabajo. Por esta razón, los indicadores basados en el número de citas lo que miden es el impacto, influencia o visibilidad de la investigación, que es un indicador indirecto de la calidad.

El factor de impacto (a dos años) de una revista es el número medio de citas de los trabajos publicados en los dos años naturales anteriores. Este indicador fue propuesto por Garfield para evaluar exclusivamente las revistas de investigación. Quienes defienden su aplicación en la evaluación de los investigadores, justifican este procedimiento en base a la creencia que aquellas revistas con mayores factores de impacto exigen mejores manuscritos, lo cual es en parte cierto al comparar trabajos del mismo campo cuando los factores de impacto de las revistas son significativamente diferentes. Sin embargo, la aplicación a investigadores que trabajan en distintos campos no es directa, dado que el factor de impacto depende de parámetros bibliométricos que no tienen que ver con la calidad como, por ejemplo, el número medio de referencias bibliográficas en las revistas del área. Por otra parte, las mejores revistas publican muchos trabajos de escaso 
éxito y, a la inversa, muchos trabajos de gran éxito se publican en revistas que no están en los primeros puestos de los listados por factor de impacto. Es bien conocida la regla 80/20, que indica que el $80 \%$ de las citas de muchas revistas son obtenidas por el $20 \%$ de los trabajos. Además, no existe correlación entre el factor de impacto de una revista y el número de citas de un trabajo concreto de esa revista, por lo que no puede emplearse el factor de impacto para predecir el éxito de los artículos. Como consecuencia, existe un rechazo generalizado a evaluar la calidad de un trabajo por el factor de impacto de la revista (Nature, 2005), sobre todo cuando determinar las citas reales de los trabajos está al alcance de cualquier investigador.

En la valoración de las publicaciones científicas se puede tener en cuenta, además, algunos aspectos como el número de páginas publicadas, en ocasiones normalizadas por el promedio de caracteres, y el número de autores de cada trabajo. Sin embargo, el número de páginas es significativo sólo cuando se consideran artículos publicados en revistas de gran prestigio (blue ribbon o top journals), en las que la extensión de los artículos refleja el esfuerzo realizado por los investigadores. Además, es conocido que existen hábitos de publicación distintos en los diferentes campos de especialidad, y que algunas revistas establecen límites estrictos al número de páginas de cada artículo, mientras que otras no lo hacen.

\subsection{Indicadores tipo $b$}

Como se ha comentado, el procedimiento para valorar la excelencia de un investigador, sin recurrir a la evaluación por pares, consiste en el análisis de la frecuencia de las citas de sus trabajos. La suma de todas las citas recibidas por los trabajos publicados por un autor refleja, en muchos casos, el éxito de su carrera científica. Sin embargo, en ocasiones, algunos éxitos puntuales acumulan un alto porcentaje del total de citas de un investigador. Además, las grandes colaboraciones introducen sesgos muy importantes, al recoger muchas citas pero derivadas del trabajo de un gran número de investigadores.

Para corregir estos sesgos, Hirsch propone un nuevo indicador. Un investigador tiene un índice $b$ cuando $b$ de sus publicaciones han recibido al menos $b$ citas cada una, y el resto tiene $b$ o menos citas por trabajo (Hirsch, 2005). Este indicador estima el número de trabajos importantes publicados por un investigador, incrementando la exigencia a la vez que aumenta su valor, y proporciona una cota inferior $b^{2}$ del número total de citas $N_{c}$. El valor de $N_{c}$ es generalmente mucho mayor que $b^{2}$ (entre 3 y 5 veces según ha estimado el propio Hirsch) ya que esta cantidad subestima las citas de los artículos más citados e ignora los artículos con menos de $h$ citas. Hirsch argumenta que $N_{c}$ puede ser inflado por un pequeño número de importantes éxitos que podrían deberse al efecto de la coautoría. Sin embargo, el índice $b$ es robusto, en el sentido que no se ve afectado ni por aquellos artículos no citados (o poco citados) ni por uno o varios artículos anormalmente muy citados. 
El índice $b$ ha sido extensamente estudiado (ver revisión de Bornmann y Daniel, 2007) y tiene importantes propiedades matemáticas (Glänzel, 2006). Sin embargo, también presenta importantes limitaciones, algunas de las cuales se indican a continuación, junto con algunas variantes que han surgido en los últimos años para intentar resolverlas.

- Depende del área y del número de colaboradores (es size-dependent). No es adecuado, por tanto, para comparar investigadores de diferentes áreas científicas, lo que se explica por los distintos hábitos de publicación y citación según el campo. El número de citas depende de dos parámetros bibliométricos que son diferentes entre campos y que no tienen que ver con la calidad, como son el promedio de referencias y autores por trabajo. El primero de estos sesgos puede corregirse dado que el $b$ máximo en cada campo está fuertemente correlacionado con los factores de impacto de las primeras revistas del área, lo que permite estimar un $b$ de referencia en cada especialidad (Imperial y Rodríguez-Navarro, 2007). Una alternativa es el índice $b$ (Bornmann y otros, 2007) que indica el número de artículos incluidos en el 10\% de los más citados en su campo, determinados a partir de los percentiles ISI-ESI, por ejemplo. El segundo sesgo puede reducirse con un indicador complementario $b_{1}$, que se obtiene al dividir por el número medio de autores de esos artículos (Batista y otros, 2006), e indicaría el número de artículos que un investigador podría haber publicado en solitario a lo largo de su carrera con al menos $b_{1}$ citas.

- Correlaciona con el número de publicaciones. Muestra una alta correlación positiva con el número total de citas y de documentos de los investigadores (Cronin y Meho, 2006; Saad, 2006; Van Raan, 2006), por lo que tiende a favorecer a los que cuentan con carreras científicas más dilatadas y tiene menos validez entre aquellos con un bajo número de publicaciones. Para diferenciar entre investigadores activos e inactivos y poder comparar investigadores en distintas etapas de su carrera, se ha propuesto la tasa de crecimiento $b^{\prime}(t)$ (Rousseau y Ye, 2008). A diferencia de $N_{c}(t)$, que es una función cuadrática, Hirsch (2007) ha estimado empíricamente que $h(t)=a \cdot t$ es una función lineal del tiempo. De esta manera, es posible tomar como elemento de comparación la tasa de crecimiento $a=b / t$, siendo $t$ los años transcurridos desde la publicación del primer artículo. Una alternativa es calcular el índice $h$ para un determinado período de tiempo, en lugar de toda la vida profesional de un investigador.

- No considera todas las citas de los artículos más citados (bighly cited papers). Estos trabajos contribuyen al índice $b$ de un determinado autor, pero el número de veces que son citados no influye sobre su valor. Esto es debido a que al estimar el éxito de un investigador por el índice $b$ se desprecian las colas de la distribución de citas. Estas colas corresponden a aquellas publicaciones que se alejan del impacto promedio, ya sea porque han sido muy citadas (cola superior), o porque apenas lo han sido (cola inferior). El 
índice $g$ (Egghe, 2006) considera todas las citas de los g artículos más citados, y viene a representar un promedio de citas entre estos $g$ artículos. Ordenados los artículos de forma decreciente del número de citas que reciben, $g$ es el mayor valor de forma que los primeros $g$ artículos tienen, en su conjunto, al menos $g^{2}$ citas. Sin embargo, como indica Hirsch, una cola superior pesada puede corresponder a trabajos con muchos autores en grandes líneas de investigación que generan muchas citas. Una cola inferior pesada indica un gran número de publicaciones de escaso impacto, lo que podría indicar sobreproducción.

- Perjudica a los investigadores selectivos, aquellos con producción moderada pero de gran impacto, frente a los grandes productores, aquellos con una gran producción pero de impacto moderado (Costas y Bordons, 2007a,b). Además, no es consistente (Waltman y Van Eck, 2009), debido a que el efecto de la incorporación de un nuevo trabajo con un número determinado de citas puede ser diferente entre investigadores, incrementando el valor de $h$ en unos casos y dejándolo igual en otros. En este trabajo se proponen los indicadores de entorno, un complemento del índice $b$ que trata de aumentar la consistencia del indicador sin favorecer a los grandes productores frente a los selectivos.

\section{Indicadores de entorno}

\subsection{Formulación matemática}

Dadas las publicaciones de un autor en orden decreciente del número de citas, sea $N_{p}$ el número total de publicaciones de un autor y $c_{i}$ el número de citas recibidas por la publicación $i\left(c_{1} \geq c_{2} \geq \ldots \geq C_{N_{p}}\right)$. Sea $N_{c}^{j}=\sum_{i=1}^{j} c_{i}$ las citas agregadas de las $j$ publicaciones más citadas, $N_{c}=\sum_{i=1}^{N_{p}} c_{i}$ las citas totales y $n_{c}=N_{c} / N_{p}$ el promedio de citas por artículo.

La representación del número de citas frente a la posición en la lista $\left(i, c_{i}\right)$ da lugar a la distribución de citas, mientras que la línea que une estos puntos es la curva de citas. El índice es el mayor número entero que cumple $c_{b} \geq b$, es decir,

$$
h=\operatorname{máx}\left\{i \in Z: c_{i} \geq i\right\}
$$

Geométricamente, es la parte entera del punto de intersección de la curva de citas con la bisectriz del primer cuadrante. Esto significa que el autor tiene $b$ trabajos con al menos $b$ citas cada uno.

El índice $b$ proporciona una cota inferior $H=b^{2}$ del número total de citas, no incluyendo el volumen de citas en las colas de la distribución de citas: cola superior " $U$ " de artículos más citados y cola inferior " $L$ " de artículos menos citados. Obsérvese que se tienen las siguientes relaciones: 


$$
\begin{aligned}
N_{c} & =H+U+L, \\
U & =N_{c}^{b}-H, \\
L & =N_{c}-N_{c}^{b}
\end{aligned}
$$

El peso relativo de las colas de la distribución de citas viene dado por $N_{c} / H$. De acuerdo a las estimaciones de Hirsch, podemos decir que si $N_{c} / H<3$ la distribución tiene colas poco pesadas, mientras que si $N_{c} / H>5$ la distribución tiene colas muy pesadas. El índice $b$ beneficia a aquellos investigadores que presentan colas menos pesadas. Esto es debido a que a medida que aumenta el peso relativo de la cola, la proporción de citas consideradas en el cálculo de este índice es menor. Perjudica de manera considerable a aquellos investigadores con una relación de colas $U / L$ elevada. Cuando $U / L>1$, el peso de la cola superior es mayor que el de la cola inferior, lo que podría indicar que se trata de un investigador selectivo. Cuando sucede lo contrario, esto es $U / L<1$, la relación entre las colas podría indicar que nos encontramos ante un investigador gran productor.

El índice $g$ es el mayor número entero que cumple $g^{2} \leq N_{c}^{g}$, es decir,

$$
g=\operatorname{máx}\left\{i \in Z / \frac{N_{c}^{i}}{i} \geq i\right\}=\operatorname{máx}\left\{i \in Z / N_{c}^{i} \geq i^{2}\right\}
$$

Este indicador considera todas las citas de los $g$ artículos más citados y representa un promedio de citas entre estos $g$ artículos. En este caso, se incluye la cola superior de citas $U$, por lo que $g \geq b$ y garantiza una cota inferior de citas $G=g^{2} \geq H$.

La mediana " $m$ " de la distribución de citas corresponde a aquella publicación que deja a cada lado aproximadamente la mitad de las citas, es decir,

$$
m=\operatorname{mín}\left\{i \in Z / N_{c}^{i} \geq \frac{N_{c}}{2}\right\}
$$

La tabla I resume la notación empleada y describe algunos indicadores bibliométricos en función del porcentaje de citas representadas. La tabla II y la figura 1 muestran, para dos ejemplos, diferentes medidas que describen la forma de la curva de citas. Además, estos ejemplos ponen de manifiesto que el índice $b$ correlaciona menos con el juicio de los expertos en Ciencias Sociales que en otras Ciencias Experimentales.

En general, los índices $b$ en Ciencias Sociales son muy inferiores a los de otras Ciencias. Así, mientras en Biología y Biomedicina el $b$ máximo es del orden de 150, en Física es del orden de 100 (Hirsch, 2005) y en Economía de 50. Como se ha indicado en el apartado 2, el $b$ máximo en cada campo está fuertemente correlacionado con los factores de impacto de las primeras revistas del área, lo que permite estimar un $h$ de referencia en cada especialidad $h_{R}=16+11 \cdot f$, donde $f$ es el factor de impacto promedio de las primeras revistas del área (Imperial y Rodríguez-Navarro, 2007). La categoría "Economics" del JCR 2007 incluye 


\section{TABLA I}

Notación y descripción de algunos indicadores bibliométricos

\begin{tabular}{|c|c|c|c|}
\hline Indicador & Descripción & Citas representadas & $\%$ de citas \\
\hline$N_{p}$ & $\mathrm{~N}^{\mathrm{o}}$ de publicaciones & 一 & 一 \\
\hline$i=1, \ldots, N_{p}$ & $\begin{array}{c}\text { Índice publicación en orden } \\
\text { decreciente del } n^{\circ} \text { de citas }\end{array}$ & - & - \\
\hline$c_{i}$ & Citas publicación $i$ & 一 & 一 \\
\hline$N_{c}^{j}=\sum_{i=1}^{j} c_{i}$ & Citas agregadas hasta $j$ & - & - \\
\hline$N_{c}^{b}$ & Citas de los $h$ más citados & $H+U$ & $\frac{H+U}{N_{c}} 100$ \\
\hline$N_{c}=N_{c}^{N_{p}}$ & Total citas & $H+U+L$ & 100 \\
\hline$n_{c}=\frac{N_{c}}{N_{p}}$ & Promedio citas por publicación & - & - \\
\hline$H$ & Cuadrado de citas de Hirsch & $b^{2}$ & $\frac{b^{2}}{N_{c}} 100$ \\
\hline$U$ & Cola superior más citados & $N_{c}^{b}-H$ & $\frac{N_{c}^{b}-H}{N_{c}} 100$ \\
\hline$L$ & Cola inferior menos citados & $N_{c}-N_{c}^{b}=N_{c}-H-U$ & $\left(1-\frac{N_{c}^{b}}{N_{c}}\right) 100$ \\
\hline G & Cuadrado de citas de Egghe & $g^{2}$ & $\frac{g^{2}}{N_{c}} 100$ \\
\hline$U+L$ & Total colas & $N_{c}-H$ & $\left(1-\frac{H}{N_{c}}\right) 100$ \\
\hline$b$ & Índice $b$ & $H$ & $\frac{H}{N_{c}} 100$ \\
\hline$g$ & Índice $g$ & $G$ & $\frac{G}{N_{c}} 100$ \\
\hline$m$ & Mediana & $\frac{1}{2} N_{c}$ & 50 \\
\hline$\frac{U}{L}$ & Relación de colas & - & - \\
\hline$\frac{N_{c}}{H}$ & Peso relativo de las colas & - & - \\
\hline
\end{tabular}

191 revistas, que presentan grandes diferencias entre factores de impacto. Aunque el mayor es 4,190, tan sólo doce superan el 2, por lo que se ha optado por tomar la media de los factores de impacto de las doce primeras revistas de la categoría, cuyo resultado es 2,958, para el cálculo del $b$ de referencia internacional. Para el cálculo del $b$ de referencia nacional se ha tomado la única revista española 


\section{TABLA II}

Indicadores bibliométricos de J. E. Stiglitz y E. S. Phelps según Web of Science (octubre, 2008)

\begin{tabular}{|c|c|c|c|c|}
\hline \multirow{2}{*}{ Indicador } & \multicolumn{2}{|c|}{ J. E. Stiglitz } & \multicolumn{2}{|c|}{ E. S. Phelps } \\
\hline & Valor & $\%$ citas & Valor & $\%$ citas \\
\hline$N_{p}$ & 164 & - & 58 & - \\
\hline$N_{c}$ & 12.016 & 100 & 2.125 & 100 \\
\hline$n_{c}$ & 73,27 & - & 36,64 & - \\
\hline$b$ & 50 & - & 20 & - \\
\hline$g$ & 108 & - & 46 & - \\
\hline$m$ & 9 & 50 & 5 & 50 \\
\hline$N_{c}^{b}$ & 10.132 & 84,3 & 1.982 & 93,3 \\
\hline$H$ & 2.500 & 20,8 & 400 & 18,8 \\
\hline$U$ & 7.632 & 63,5 & 1.582 & 74,4 \\
\hline$L$ & 1.884 & 15,7 & 143 & 6,7 \\
\hline$G$ & 11.664 & 97,1 & 2.116 & 99,6 \\
\hline$U+L$ & 9.516 & 79,2 & 1.725 & 81,2 \\
\hline$\frac{U}{L}$ & 4,05 & - & 11,06 & - \\
\hline$\frac{N_{c}}{H}$ & 4,81 & - & 5,31 & - \\
\hline
\end{tabular}

FIGURA 1

Distribución de citas de J. E. Stiglitz y E. S. Phelps según Web of Science (octubre, 2008)

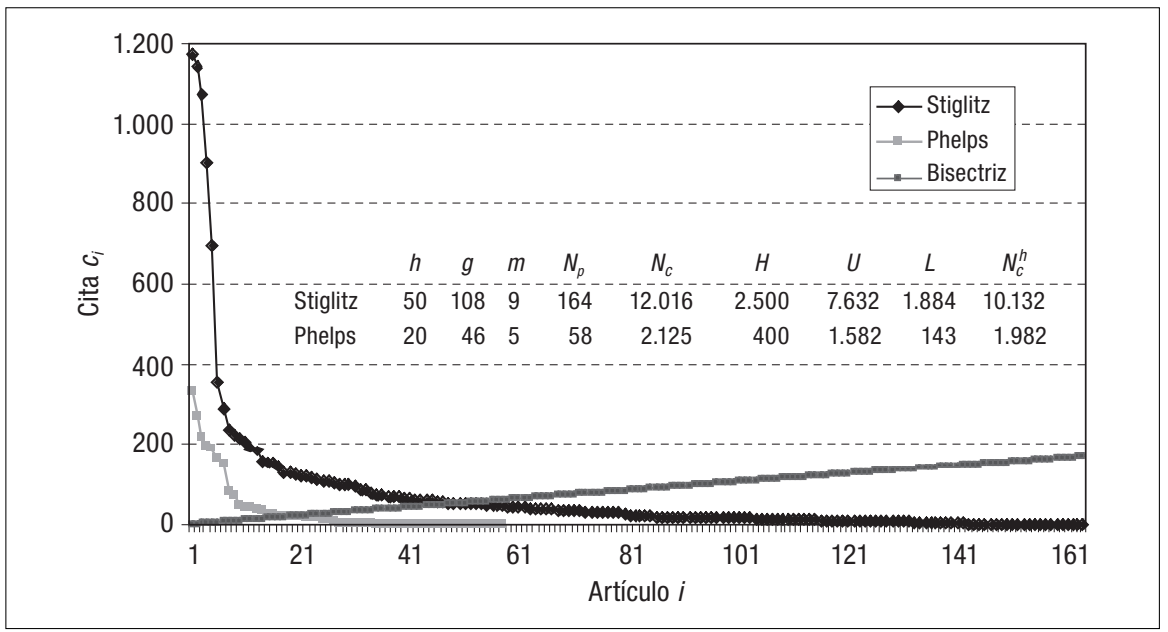


en esta categoría (Investigaciones Económicas) cuyo factor de impacto es 0,268. Estos datos indican que el índice de referencia internacional en Economía es del orden de $50\left(h_{R}=16+11 \cdot 2,958 \simeq 49\right)$, mientras que en España es del orden de $20\left(h_{R E}=16+11 \cdot 0,268 \simeq 19\right)$.

Aunque algunos economistas de prestigio, atendiendo al juicio de expertos, tienen índices entorno al de referencia (por ejemplo, Joseph E. Stiglitz, premio Nobel en 2001 y $b=50$ ), los índices de otros economistas de prestigio son muy inferiores (por ejemplo, Edmund S. Phelps, Nobel en 2006 y $h=20$ ). Estos ejemplos, que se describen en la tabla II y la figura 1, ilustran que el índice $b$ en algunas disciplinas sociales correlaciona menos con el juicio de expertos, siendo necesario complementarlo con otros indicadores.

\subsection{El problema de discriminar entre índices similares}

Al comparar las carreras profesionales de dos científicos de un mismo campo, surgen dudas en aquellos casos en los que las curvas de citas se cortan. Las figuras 2 y 3 muestran dos ejemplos en los que esto sucede. En ambos casos, el

FIGURA 2

Dos curvas de citas que se cortan, con idéntico índice y número total de citas, pero diferente promedio de citas por artículo

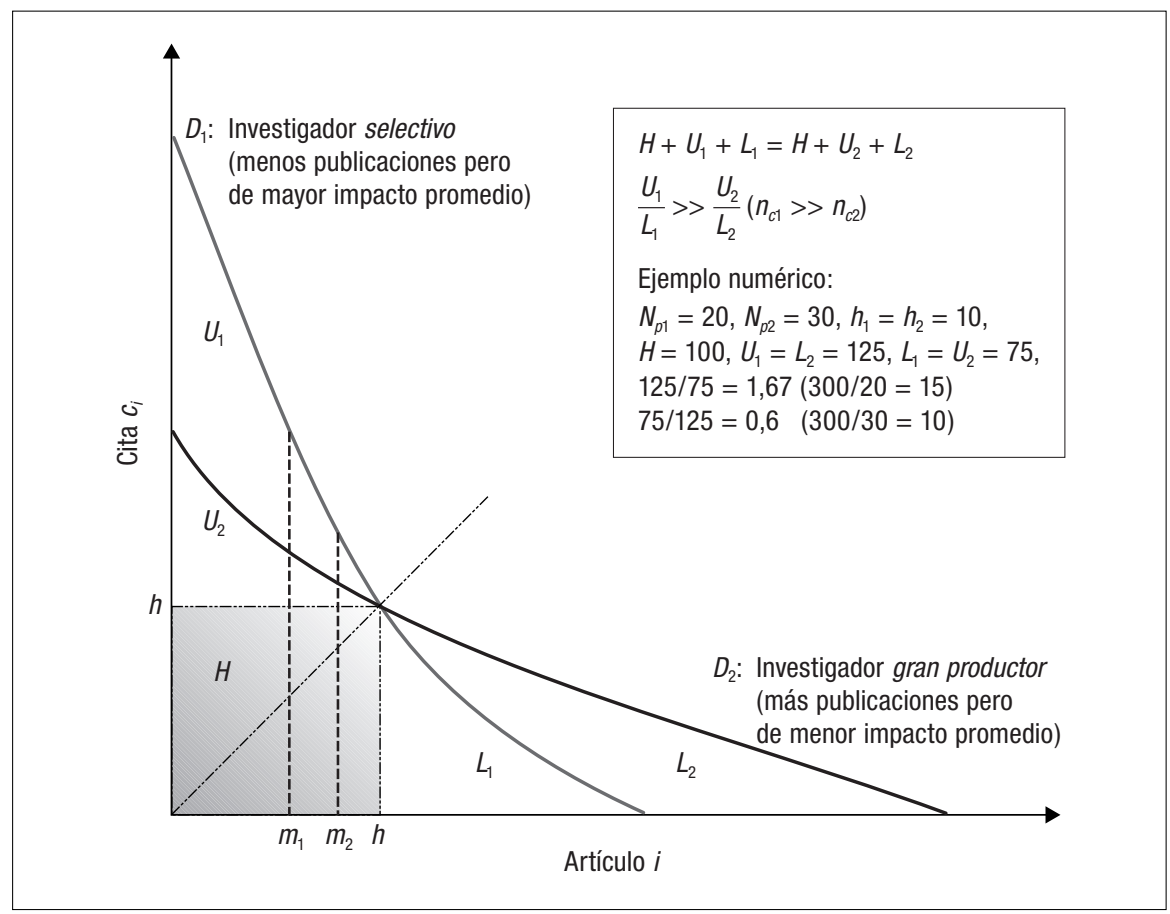




\section{FIGURA 3}

Dos curvas de citas que se cortan, donde la de menor índice «b" presenta mayor promedio de citas por artículo

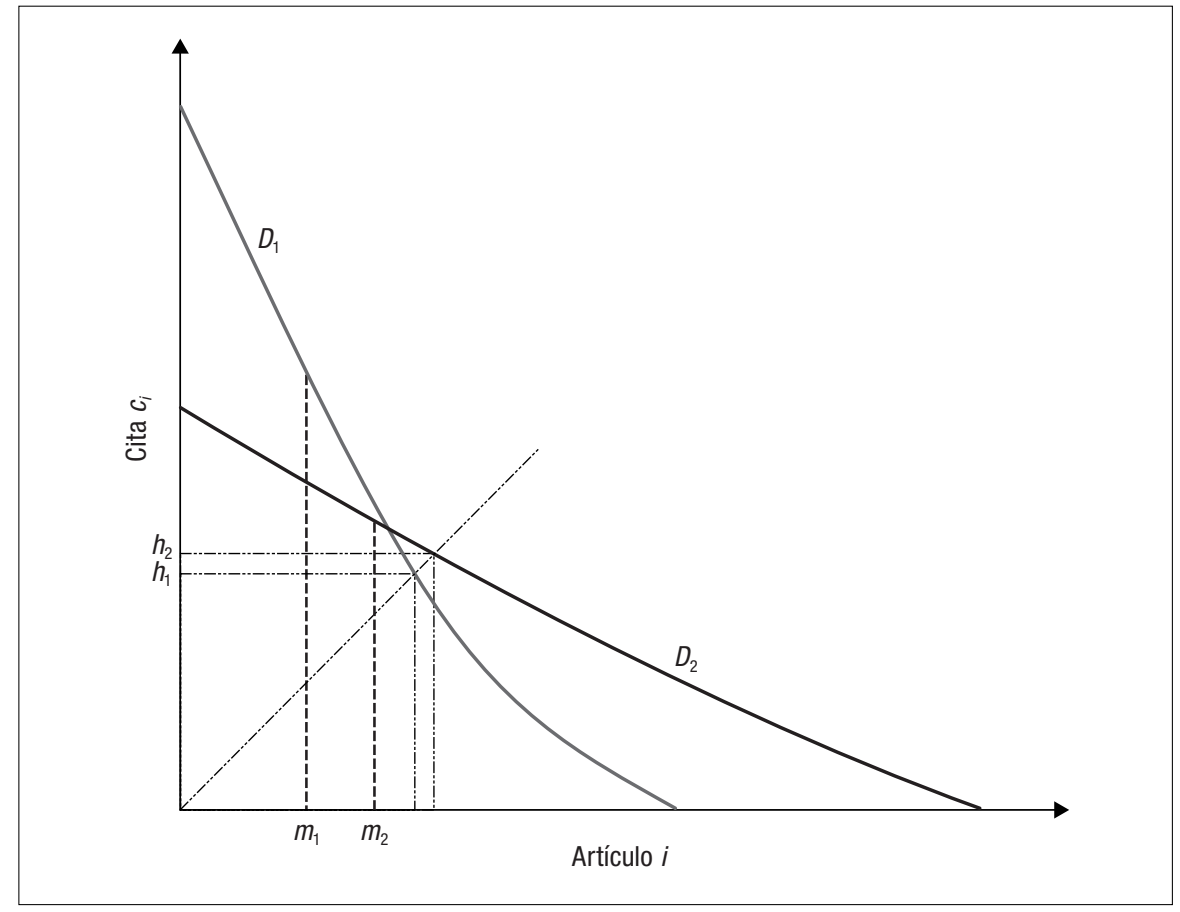

primer investigador presenta niveles más altos de citas al comienzo de la curva y más bajos al final, pero ¿qué gráfica sugiere una carrera más consolidada y constante? En la figura 2 se aprecian dos perfiles muy diferentes entre investigadores, uno selectivo frente a otro más masivo en la publicación, sin embargo, ambos presentan idéntico índice $h$. Como muestra la figura 3, el índice $b$ de un investigador puede incluso ser menor al del otro, aunque eso no tiene porqué indicar necesariamente que el primero presente una carrera menos sólida. Estas gráficas reflejan el problema de discriminar entre dos distribuciones con similares indices $h$ (no significativamente diferentes) pero con diferencias importantes en la relación entre las colas de la distribución $U / L$.Como se puede observar, a mayor ratio de colas, mejor promedio de citas por artículo.

La mediana divide la distribución de citas en dos partes con similar número de citas, y cuando la distribución es simétrica respecto a la bisectriz, se tiene $m<b$ y $U=L$. El caso $m<<b$ (donde $<<$ indica que es mucho menor) corresponde a una distribución donde $U>>L$, mientras que $m>>b$ corresponde a $U$ $\ll L$. El índice $b$ no discrimina entre perfiles diferentes dado que no tiene en cuenta las colas de la distribución, ni siquiera para valores de $m$ muy alejados 
de $h$. Podría darse el caso de dos distribuciones con el mismo $h$, una con $U>>L$ y otra con $U<L$. Parece razonable, en estos casos, medir de alguna manera parte de $U$ y $L$ para complementar el índice $b$ con las zonas próximas al cuadrado $H$ de citas de Hirsch. De esta forma, se incrementa la capacidad de discriminación en aquellos casos en los que existan dudas razonables entre investigadores. Esta idea da lugar a los indicadores de entorno.

\subsection{Indicadores de entorno $H_{j}, j=0,1, \ldots, b-1$}

Sea $A(B)$ la zona superior (inferior) contigua a $H$, esto es, aquella parte de la cola superior $U$ (inferior $L$ ) de la distribución de citas más próxima al cuadrado de citas de Hirsch. La zona inferior $B$ corresponde a aquellos artículos que con mayor probabilidad pueden contribuir a incrementar el valor de $b$ en el futuro, dado que están más próximos al núcleo que define su valor. La zona superior $A$ incluye aquellas citas que formarán parte del cuadrado $H$ en el momento en que el índice $b$ aumente de valor. Por ello, parece razonable relajar la restricción para incluir también dichas zonas y aumentar, de esta forma, el poder de discriminación del indicador.

Los indicadores de entorno son una generalización del cuadrado $H$ de citas de Hirsch, que considera estas zonas contiguas $(A$ y $B$ ) en cierto entorno de $b$ de la forma $h \pm j, 0,1, \ldots, b-1$, y están definidos de la siguiente manera:

$$
H_{j}=H_{b \pm j}=(b-j) c_{h-j}+\sum_{i=h-j+1}^{b+j} c_{i}, j=0,1, \ldots, b-1
$$

Obsérvese que $H_{0}=H$, por lo que este indicador generaliza el cuadrado de citas de Hirsch. El último indicador es $H_{b-1}=N_{c}^{2 b-1}$, que incluye toda la cola superior de citas $U$. Aunque podría definirse el indicador también para valores $\geq h$, hay que tener en cuenta que, en tal caso, sólo estaríamos añadiendo parte de la cola inferior $L$.

La figura 4 muestra los indicadores de entorno para dos distribuciones de citas. Como puede observarse, en el caso de la distribución $i, i=1,2$, el indicador de entorno agrega el área $A_{i}+B_{i}$ al cuadrado $H$.

Dado que el indicador de entorno representa un agregado de citas, su representación en función del radio del entorno es una función creciente. Esto puede observarse en la figura 5 , donde se muestra el comportamiento de este indicador para J. E. Stiglitz y E. S. Phelps hasta $j=19$, que corresponde al último entorno para el autor con menor índice $h$.

Aquellos autores cuyas distribuciones de citas presentan colas pesadas son perjudicados por el índice $h$. Sin embargo, para estos autores el indicador de entorno crece más rápidamente que para los investigadores con colas menos pesadas, aumentando el poder de discriminación. Los investigadores selectivos también son perjudicados por el índice $h$. Sin embargo, el indicador de entorno corrige este problema. A modo de ejemplo, supongamos un investigador con 


\section{FIGURA 4}

Indicadores de entorno para dos curvas de citas con idéntico índice

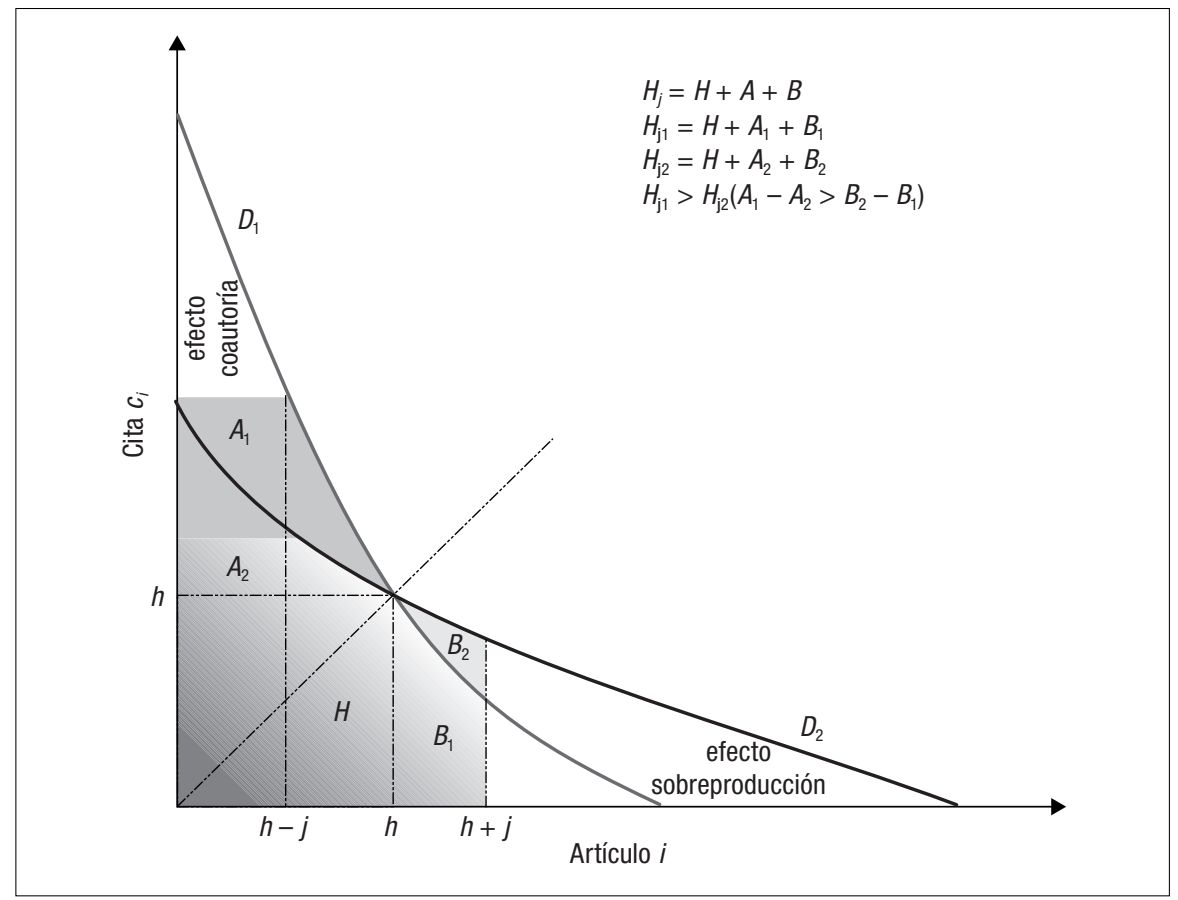

FIGURA 5

Indicadores de entorno para J. E. Stiglitz y E. S. Phelps según Web of Science (octubre, 2008)

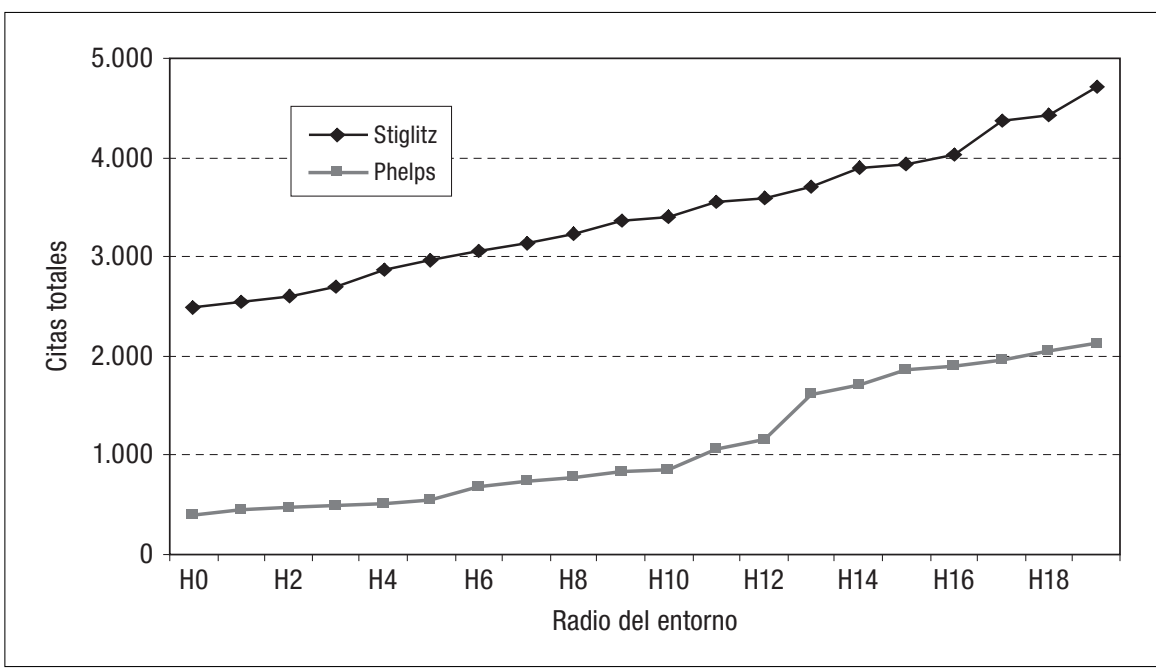


10 publicaciones, la menos citada de las cuales tiene 20 citas. En este caso $b=10$ y representa tan sólo a 100 de las citas. Sin embargo, $H_{1}=200$ por lo que se ha doblado el volumen de citas representadas sin más que considerar un entorno de radio 1 .

Una fórmula recurrente, más eficiente que la anterior a la hora de programar el cálculo de los indicadores de entorno, es:

$$
\begin{aligned}
& H_{0}=b^{2}, \\
& H_{j}=H_{j-1}+\left(c_{b-j}-c_{b-j+1}\right)(b-j)+c_{b+j}, \quad j=1, \ldots, b-1
\end{aligned}
$$

En este trabajo se ha optado por considerar entornos de centro $b$ y radio $j$, aunque de forma similar se podrían definir indicadores de intervalo, y considerar las citas de las zonas próximas atendiendo a cierto intervalo de la forma $[h-\alpha$, $b+\beta$, resultando el conjunto de indicadores:

$$
H_{b-\alpha, b+\beta}=(b-\alpha) c_{h-\alpha}+\sum_{i=h-\alpha+1}^{b+\beta} c_{i}, \quad 0 \leq \alpha \leq b-1, \quad 0 \leq \beta \leq N_{p}-b
$$

Cuando $\alpha=\beta=0$, se obtiene $H$; para $\alpha=b-1, \beta=0$, se obtiene $N_{c}^{h}$; para $\alpha=b-1, \beta=N_{p}-b$, se obtiene $N_{c}$; y, finalmente, cuando $\alpha=\beta=j$ se tiene el indicador de entorno $H_{j}$.

En aquellos casos en los que $h$ es grande, se podría tomar, por ejemplo, $\alpha=\beta=[h / 2]$, siendo $[x]$ la parte entera de $x$, resultando el intervalo $\left[\frac{b}{2}, \frac{3 h}{2}\right]$ en el caso particular en que $b$ es un número par. Sin embargo, cuando $b$ es pequeño podría ser más razonable considerar, por ejemplo, $\alpha=\left[\frac{b}{2}\right], \beta=h$, resultando el intervalo $\left[\frac{b}{2}, 2 b\right]$ en el caso particular en que $b$ es un número par.

\section{Ejemplo}

Se ha estudiado el comportamiento de los indicadores de entorno en el conjunto de autores españoles en activo más productivos en Ciencias Sociales. Para ello, se considera la distribución de citas de una muestra aleatoria de $n=16$ autores de la población TOP-100, formada por los 100 investigadores españoles con mayor número de artículos en la base de datos ISI-SSCI a diciembre de 2008, que cuentan con alguna publicación durante el año 2008. En la población TOP-100 todos los autores tienen al menos 28 artículos. La muestra tomada contiene 901 artículos de los 4064 que conforman la población en su conjunto, aproximadamente un $22 \%$.

El objetivo es obtener los indicadores de entorno $H_{j}, j=0,1, \ldots, b-1, \mathrm{y}$ estimar empíricamente el valor de $j$ que más correlaciona con el $H$ futuro. Para 
ello, se ha tomado el número de artículos y citas a diciembre de 2003 y 2008. Además, con la intención de ver el efecto que pudiera tener el índice $b$ sobre los indicadores de entorno, se han considerado dos grupos atendiendo a los datos de 2003, el primero formado por aquellos autores de la muestra con $b \leq 7$ (grupo 1) y el segundo con el resto de autores, es decir, aquellos con $b \geq 8$ (grupo 2).

La tabla III muestra, para cada autor, el rango de publicaciones, con el año del primer artículo en la base de datos SSCI, y el total de artículos y citas en 2003 y 2008. Esta información se muestra también en la figura 6, cuya figura a) permite visualizar aquellos autores con mayor impacto (Alonso y Lobo). Las figuras $b$ ) y c) de este gráfico muestran los diagramas de dispersión producciónimpacto, de tal manera que aquellos autores por encima de la recta de regresión muestran un comportamiento más selectivo que aquellos situados por debajo, que serían menos selectivos. Como puede observarse, aunque existen diferencias significativas en la relación producción-impacto de ambos gráficos (2003 y 2008), en ambos casos los autores más selectivos de la muestra son Alonso, Lobo y Tobena (9, 12 y 15, respectivamente) y el menos selectivo es Gil-Alana (3). Este autor ha publicado en 9 años un total de 65 trabajos que han recibido 142 citas, lo que hace un promedio de 2,18 citas por artículo. Todo lo contrario sucede con Lobo, por ejemplo, ya que en 31 años ha publicado 62 trabajos que han recibido 1.505 citas, una media de 24,27 citas por artículo.

La tabla III muestra también el índice $h$ en $2003, h_{2003}$, y en $2008, h_{2008}$, y los indicadores de entorno para 2003, $H_{j}, j=0,1,2$, 3. Aunque se ha tomado una muestra de los investigadores más productivos, en seis de los casos $b \leq 3$, por lo que para $j=3$ sólo se dispone de 10 datos. Finalmente, este cuadro muestra también la correlación lineal y log-lineal entre los indicadores de entorno y $H_{2008}$ para los dos grupos estudiados, así como para el total de autores. Como puede observarse, las correlaciones son altas en la mayoría de los casos, y tienden a aumentar a medida que crece el entorno. Comparando las correlaciones por grupos de autores, puede observarse como para el conjunto de autores con menor índice $h$ (grupo 1) el modelo log-lineal se ajusta mejor a los datos, siendo las correlaciones mayores en todos los casos analizados. Sin embargo, para el conjunto de autores con mayor $h$ (grupo 2) el modelo lineal tiene mejor ajuste en todos los casos.

A modo de ejemplo, veamos dos casos donde se pone de manifiesto la capacidad de discriminación de los indicadores de entorno frente al índice $b$. Matute y Vieta tienen $b_{2009}=9$ pero, sin embargo, todos los indicadores de entorno calculados son mayores para el segundo autor. Atendiendo a estos indicadores, sería de esperar un $h_{2008}$ mayor para Vieta, lo cual es cierto (23 frente a 13). En el caso de Calvo y Salamero sucede algo parecido. Ambos autores tienen $b_{2003}=10$ y todos los indicadores de entorno son también mayores para el segundo autor. Esto indicaría mayor $h_{2008}$ para Salamero, lo cual es también cierto (19 frente a 16). En los dos ejemplos anteriores, no sería posible discriminar entre los investigadores basándonos exclusivamente en el índice $h$. Sin embargo, los indicado- 


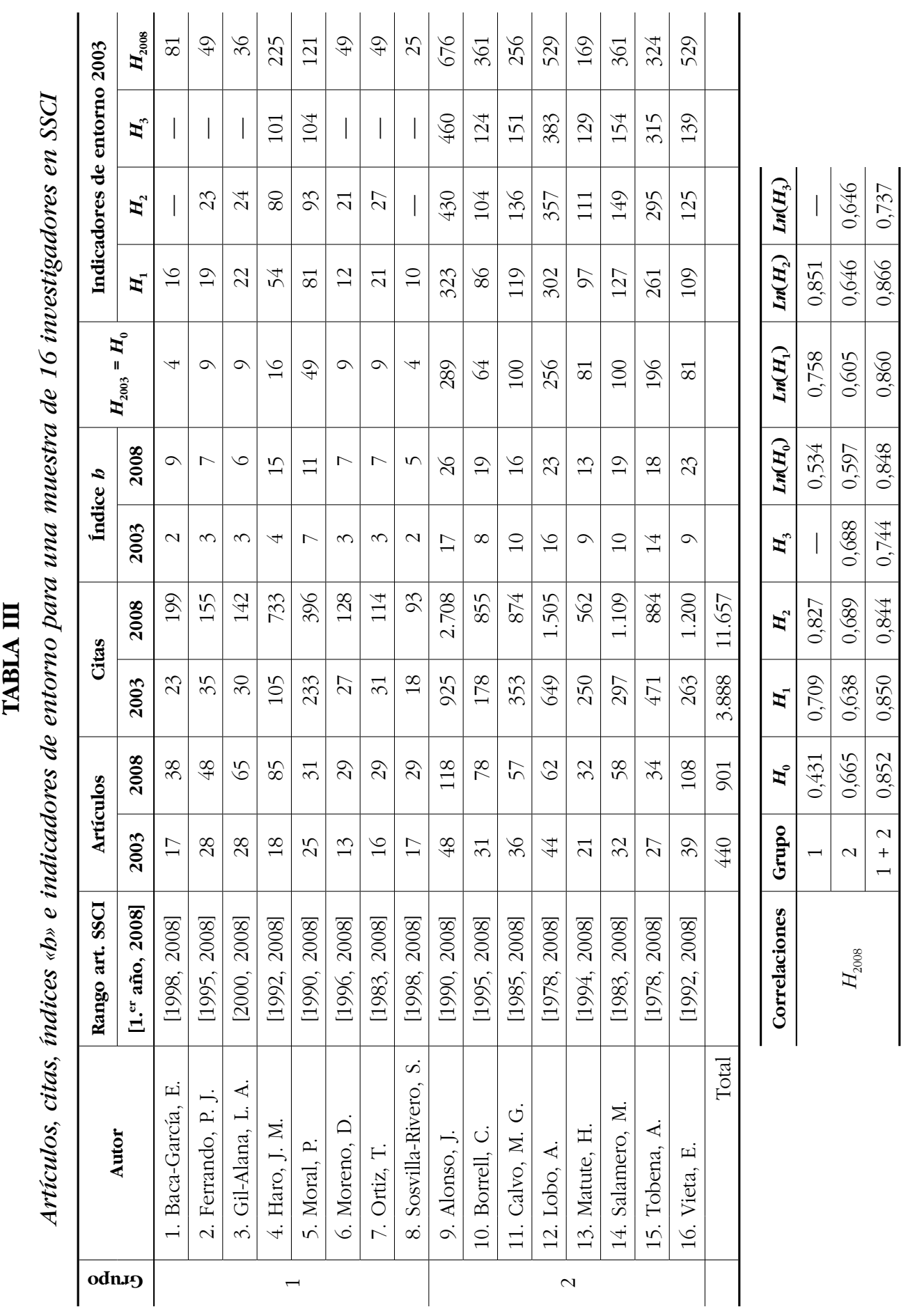


FIGURA 6

Impacto y diagramas de dispersión producción-impacto para una muestra de 16 investigadores en SSCI

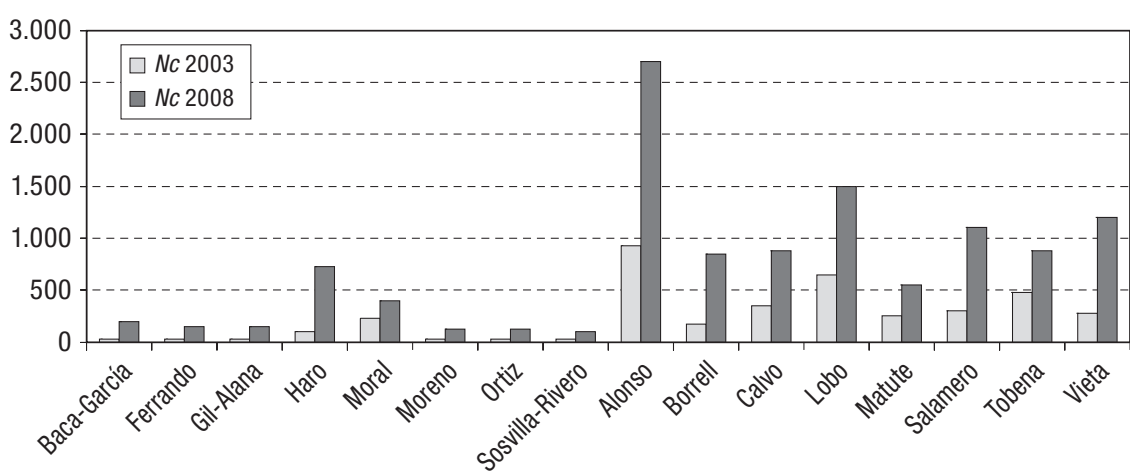

a)

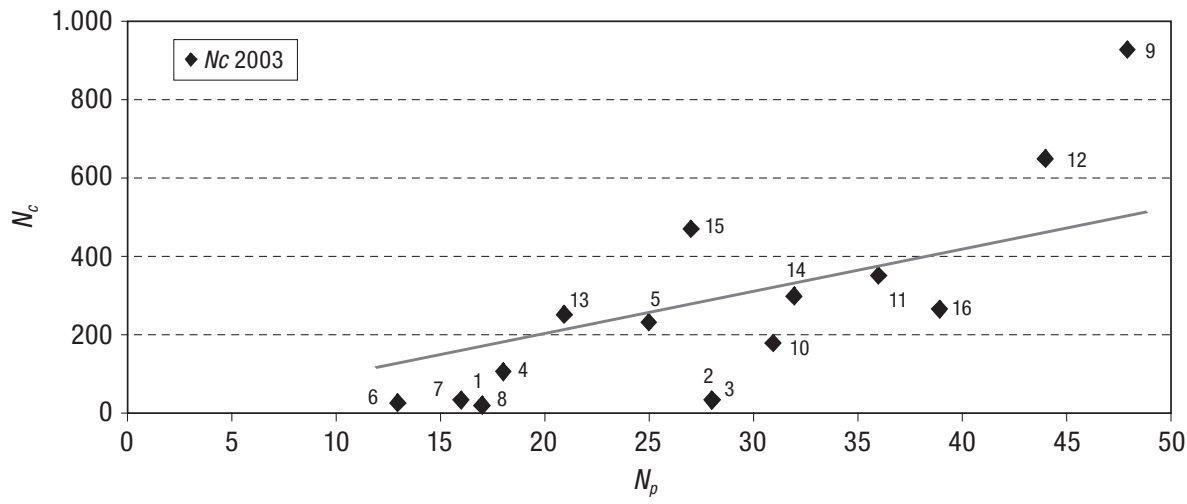

b)

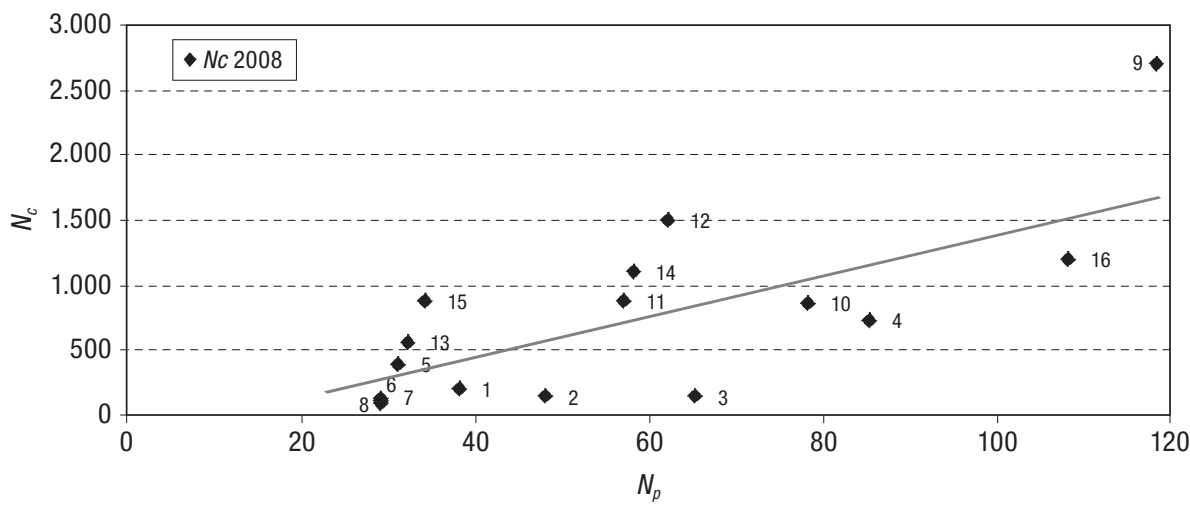

c) 


\section{FIGURA 7}

Diagramas de dispersión para una muestra de 16 investigadores en SSCI

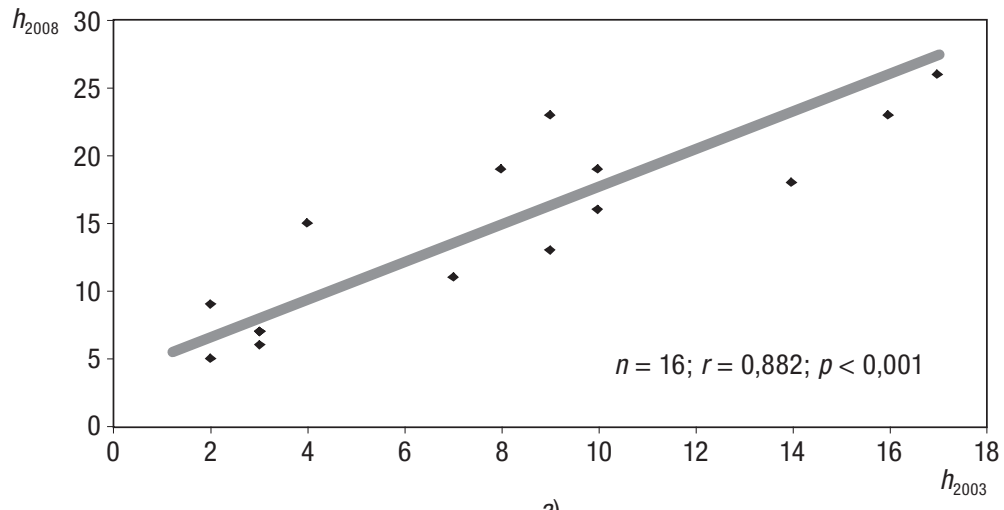

a)

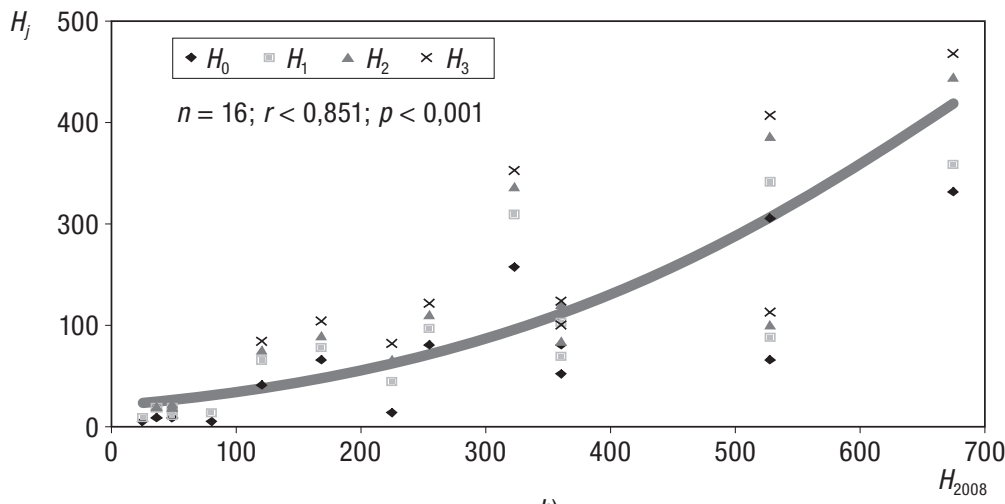

b)

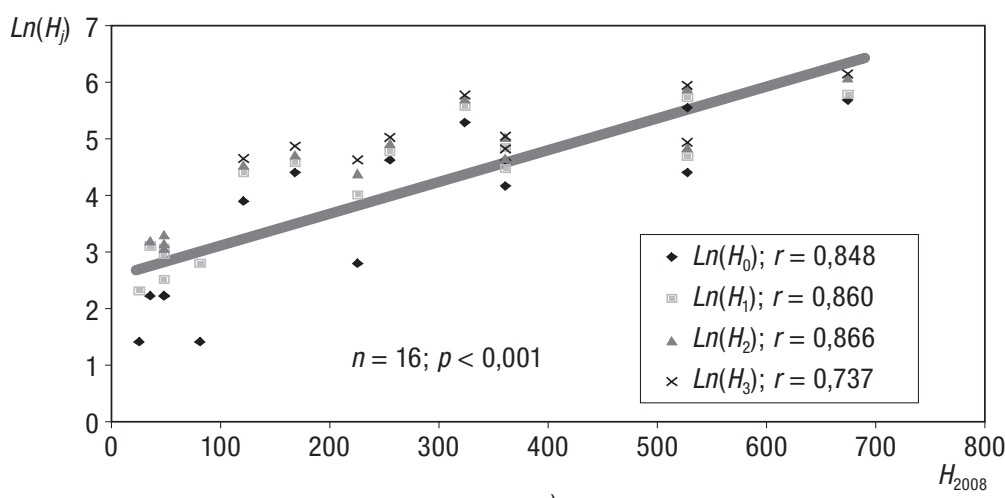

c) 
res de entorno reflejan las diferencias existentes en la distribución de citas de los autores, estimando mejor el valor de $b_{2008}$.

Finalmente, en la figura 7 se observa cómo existe correlación lineal alta entre $b_{2003}$ y $h_{2008}(r=0,882)$. El coeficiente de determinación es $r^{2}=0,779$, es decir, $h_{2008}$ es explicado en un $78 \%$ por $h_{2003}$. La correlación es significativa por encima del 99\% de seguridad. También puede observarse que existe correlación no lineal (exponencial) entre $H_{j}$ y $H_{2008}$. En el modelo log-lineal todas las correlaciones son altas, significativas por encima del $99 \%$ de seguridad, y aumentan hasta $H_{2}$. Esto indica que el mejor estimador para este ejemplo es $H_{2}$. Hay que tener en cuenta que para $H_{3}$ la correlación está calculada en relación a diez datos exclusivamente, dado que el indicador no está definido para el resto de los casos.

\section{Conclusiones}

El índice $b$ es un indicador bibliométrico que trata de medir el éxito de la carrera profesional de un investigador sólo con una parte del volumen total de publicaciones y citas. El hecho de no considerar toda la producción e impacto corrige ciertas situaciones anómalas derivadas de grandes colaboraciones o éxitos puntuales, que pueden no ser significativas de la carrera profesional en su conjunto. Sin embargo, como se ha puesto de manifiesto en este trabajo, en ciertas ocasiones, dos distribuciones de citas muy diferentes pueden dar lugar a un mismo índice $h$. En estos casos, no es posible distinguir entre ambos investigadores, por ejemplo, entre un investigador con pocos trabajos muy citados y otro con muchos trabajos de escaso impacto. Además, los bajos niveles de producción y citas en áreas como las Ciencias Sociales, se traducen en índices significativamente inferiores a los de otras áreas, por lo que la cota inferior del volumen de citas garantizadas por el indicador es menor y su capacidad para discriminar se ve reducida. Esta es una de las razones por las que el índice correlaciona menos con el juicio de los expertos sociales.

En este trabajo se ha propuesto un complemento al índice $h$, los indicadores de entorno, en relación a distinguir de entre un grupo de investigadores con valores similares de $h$, aquellos con una carrera más consolidada y constante. Estos indicadores de entorno consideran, por un lado, las citas de aquellos artículos que con mayor probabilidad pueden contribuir a incrementar el valor de $b$ en el futuro, dado que están más próximos al núcleo de artículos considerados por el índice $h$, y por otro, aquellas citas que formarán parte de $b^{2}$ en el momento en que $b$ aumente de valor.

Como se ha puesto de manifiesto en el ejemplo, este indicador tiene mayor poder de discriminación al considerar un volumen mayor de citas, y corrige un problema descrito en la literatura que indica que el índice $b$ beneficia a los grandes productores frente a aquellos más selectivos. Este sesgo se ve reducido con el indicador de entorno al incluir un volumen de citas adicional mayor en el caso de los autores selectivos. 
La principal dificultad en la aplicación práctica del indicador de entorno, en la evaluación del personal investigador, radica en la necesidad de estimar adecuadamente el radio del entorno que mejor describe la forma de la distribución de citas de la población objeto de estudio. Dicho radio debe estimarse empíricamente. En el ejemplo mostrado, donde hay un grupo con $b$ relativamente bajo, este radio es de dos artículos alrededor del índice $h$. Sin embargo, entendemos que es necesaria una aplicación empírica más exhaustiva que proporcione los valores de referencia del entorno en función del $h$ de los investigadores.

Finalmente, nos gustaría puntualizar que el indicador de entorno no pretende ser sustituto sino complemento del índice $b$, especialmente en aquellos procesos de evaluación del personal investigador donde puedan existir dudas razonables.

\section{Bibliografía}

Batista, P. D.; Campiteli, M. G.; Kinouchi, O., y Martinez, A. S. (2006): Is it possible to compare researchers with different scientific interests?, Scientometrics, v. 68, $\mathrm{n}^{\circ} 1$, 179-189.

Bornmann, L., y Daniel, H. D. (2007): What do we know about the h Index?, Journal of the American Society for Information Science and Technology, v. 58, n 9, 1381-1385.

Bornmann, L.; Mutz, R., y Daniel, H. D. (2007): The b index as a measure of scientific excellence. A promising supplement to the h index?, Cybermetrics, v. 11, $\mathrm{n}^{\circ} 1$, paper 6.

Costas, R., y Bordons, M. (2007a): Una visión crítica del índice h: algunas consideraciones derivadas de su aplicación práctica, El profesional de la información, v. 16, $\mathrm{n}^{\circ}$ 5, 427-432.

Costas, R., y Bordons, M. (2007b): The h-index: advantages, limitations and its relation with other bibliometric indicators at the micro-level, Journal of Informetrics, v. 1, no 3 , 193-203.

Cronin, B., y Meho, L. I. (2006): Using the h-index to rank influential information scientists, Journal of the American Society for Information Science and Technology, v. 57, $\mathrm{n}^{\circ}$ 9, 1275-1278.

Egghe, L. (2006): Theory and practise of the g-index, Scientometrics, v. 69, n ${ }^{\circ} 1,131-152$.

Glänzel, W. (2006): "On the h-index. A mathematical approach to a new measure of publication activity and citation impact, Scientometrics, v. 67, n ${ }^{\circ} 2,315-321$.

Hirsch, J. E. (2005): An index to quantify an individual's scientific research output, Proceedings of the National Academy of Sciences, v. 102, no 46, 16569-16572.

Hirsch, J. E. (2007): Does the h index have predictive power?, Proceedings of the National Academy of Sciences, v. 104, n 49, 19193-19198.

Imperial, J., y Rodríguez-Navarro, A. (2007): Usefulness of Hirsch's h-index to evaluate scientific research in Spain, Scientometrics, v. 71, $\mathrm{n}^{\circ}$ 2, 271-282.

Nature editorial (2005): Ratings games, Nature, v. 436, n 7053, 889-890.

Rousseau, R., y Ye, F. Y. (2008): A proposal for a dynamic h-type index, Journal of the American Society for Information Science and Technology, v. 59, $\mathrm{n}^{\circ} 11,1853-1855$. 
Saad, G. (2006): Exploring the h-index at the author and journal levels using bibliometric data of productive consumer scholars and business-related journals respectively, Scientometrics, v. 69, $\mathrm{n}^{\circ} 1,117-120$.

Van Raan, A. F. J. (2006): Comparisons of the Hirsch-index with standard bibliometric indicators and with peer judgment for 147 chemistry research groups, Scientometrics, v. $67, n^{\circ} 3,491-502$.

Waltman, L., y Van Eck, N. J. (2009): A taxonomy of bibliometric performance indicators based on the property of consistency, http://repub.eur.nl/publications/index/229666761/ 